\title{
On the Origin and Behavior of Irradiation-Induced C-component Dislocation Loops in Magnesium
}

\author{
Weizong Xu ${ }^{1}$, Yongfeng Zhang ${ }^{2}$, Guangming Cheng ${ }^{1}$, Suveen N. Mathaudhu ${ }^{3}$, Ronald O. \\ Scattergood $^{1}$, Carl C. Koch ${ }^{1}$, Enrique J. Lavernia ${ }^{4}$, Yuntian $\mathrm{Zhu}^{1^{*}}$ \\ ${ }^{I}$ Department of Materials Science and Engineering, North Carolina State University, Raleigh, NC 27695, \\ USA \\ ${ }^{2}$ Fuels Modeling and Simulations, Idaho National Laboratory, Idaho Falls, ID 83415, USA \\ ${ }^{3}$ Department of Mechanical Engineering, University of California, Riverside, California 92521, USA \\ ${ }^{4}$ Department of Chemical Engineering and Materials Science, University of California-Irvine, Irvine, CA \\ 92697, USA
}

\begin{abstract}
C-component dislocation loops are one of the unique defects in hexagonal close-packed (hcp) crystals that promote the accelerated growth and void formation under irradiation. Here, we report in situ observation of c-component dislocation loop formation in $\mathrm{Mg}$ under electron irradiation with emphasis on their atomic structures. Aberration-corrected scanning transmission electron microscopy imaging is utilized to reveal four possible types of double-layer loops, which were identified as different types of stacking fault and dislocation core structures. Triple- and quadruple-layer c-component dislocation loops were also observed. The formation mechanisms of the four types of double-layer loops were revealed via molecular dynamics simulations. The experimentally observed formation rate of the single- and double-layer dislocation loops is controlled by their formation energies. Our direct experimental observations in combination with
\end{abstract}


molecular dynamics simulations provide fundamental insight into the mechanisms governing nucleation and growth of the c-component dislocation loops as well as their interactions, which could potentially help with future development of irradiation-resistant materials.

Keywords: In situ transmission electron microscopy; c-component dislocation loop; Atomic structure; Magnesium; Stacking faults

\section{Introduction}

Crystalline defects can be introduced into the materials when being bombarded with high-energy particles such as neutrons, ions, and electrons. Such bombardment causes atom displacements from their equilibrium state in the lattice, forming interstitials and vacancies or their clusters, which can be subsequently agglomerated into dislocation loops, stacking fault tetrahedra, or voids [1-9]. Irradiated-induced dislocation loops, in particular, can form in a variety of defect structures with different Burgers vectors depending on the crystal structures, irradiation dosage and impurities [10-14]. These different defect structures can become sinks for point defects and interact with each other, which determine the materials resistance to irradiation induced hardening, creep, anisotropy growth and void swelling [15-20]. Fundamental knowledge of these defect structures is thus needed in order to understand their formation, interaction, and evolution.

C-component dislocation loops are one unique type of irradiation-induced defects in hexagonal close-packed (hcp) crystals. It can be formed during neutron, electron and ion irradiation where vacancies and self-interstitial atoms (SIAs) are generated and agglomerated into 
the close-packed basal (0001) c-planes [21-27]. Consequently, a c-component dislocation loop contains $1 / 2<0001>$ Burgers vector component, and alters the stacking sequence of the perfect hcp crystal. Stacking fault structure is thus a characteristic of these c-component dislocation loops, which is similar to the case in face-centered cubic (fcc) crystals, whose irradiation induced Frank partial loop is $1 / 3<111>$. Further diffusion of point defects into the loops enlarges their sizes, but adversely raises the stacking fault energy that necessitates to be reduced. For fcc crystals, the stacking fault of the loop can be fully erased as the loop structure transforms into a perfect dislocation $(\mathbf{b}=1 / 2[110])$ via Shockley partial dislocation shear: $1 / 6[\overline{1} 2 \overline{1}]+1 / 6[2 \overline{1} \overline{1}]+1 / 3[111] \rightarrow 1 / 2[110]$. As the perfect dislocation is formed, the loop is no longer restricted to the close-packed plane and can further grow or slip away. Similarly, in hcp structure, a faulted loop of $1 / 2\langle 10 \overline{1} 0\rangle$ formed on a prism plane can also be annihilated by shear: $1 / 6[\overline{1} 2 \overline{1} 0]+1 / 2[10 \overline{1} 0] \rightarrow 1 / 3[11 \overline{2} 0][28]$

The above dislocation transformation mechanism, however, cannot be applied to the c-component dislocation loops located on the basal plane. Alternatively, the excess stacking fault energy can only be partially reduced by changing the loop structure from an extrinsic type (i.e., E type, $\mathbf{b}=1 / 2<0001>$ ) to an intrinsic one (i.e., I1 loop: $\mathbf{b}=1 / 6\langle 2 \overline{2} 03\rangle$ ). In this new configuration, interstitial/vacancy layers are retained in the c-component dislocation loops, locking the loops in the basal plane. Eventually, the accumulation of such c-component dislocation loops on a basal plane causes lattice strain in c-direction, [0001], depending upon the interstitial or vacancy nature of the loop. It has been reported that the anisotropic expansion in $\mathrm{Zr}$ under neutron irradiation could be partially attributed to c-component dislocation loop formation [29]. C-component dislocation loops were also observed in $\mathrm{Zr}$ during the onset of accelerated/breakaway growth [30, 
31]. Griffiths et al. further revealed the microstructure of $\mathrm{Zr}$ and $\mathrm{Ti}$ under electron and neutron irradiation, and found a co-existing relationship between void and c-component dislocation loop $[32,33]$. Such coexistence is recently demonstrated in the void formation in $\mathrm{Mg}$ during electron irradiation, where the void nucleation and growth is promoted near the inner edge side of the loop $[34,35]$.

The stationary nature of c-component dislocation loops on the basal plane can promote more complicated multiple-layer loop structure formation. Double-layer loop structure is commonly observed in all hep metals under irradiation, including $\mathrm{Zr}, \mathrm{Zn}, \mathrm{Cd}$ and $\mathrm{Mg}[5,11,17,21,28,31$, 36]. In quenched $\mathrm{Mg}$, double- or even quadruple-layer vacancy c-component dislocation loops were reported $[37,38]$. Considering the consequence of stacking fault removal via the formation of a double-layer loop structure, preferential formation of a new layer on the existing faulted loop is generally believed responsible for the double-layer loop formation, which leads to lower energy [5]. Such a process could also be driven by the interaction between point defects and stacking faults as a complementary mechanism [39]. Although double- or multi-layer c-component dislocation loops have been observed in many hcp materials, their atomic structural details are still not very clear. So far, incomplete types of double-layer loop configurations have been reported and inferred from the indirect diffraction contrast analysis in TEM, and multiple loop structure is even less clear [5,38]. This is largely due to the difficulties in determining the Burgers vector and the stacking fault type of loops in transmission electron microscopy (TEM) diffraction contrast imaging, particularly when loop size is small or loop edges are close to each other. This conventional TEM approach also loses the capability in revealing the dynamic process of loop structure formation as a number of specimen tilts are required to unambiguously identify 
the dislocation loop structure. High-resolution TEM (HRTEM) imaging may help with the determination, but it suffers from issues of phase contrast imaging or delocalization, causing large uncertainty in determining the stacking sequences and atomic structure of the faulted loop.

In this paper, we report an in situ observation of multi-layer c-component dislocation loops in high angle annular dark-field (HAADF) imaging in scanning transmission electron microscopy (STEM) and HRTEM. The atomic structures of c-component dislocation loops are clearly revealed utilizing aberration-corrected HAADF-STEM Z-contrast imaging. Several configurations of double- and multiple-layer loop structure are found and categorized. The observed new configurations are thought to be at the high-energy state and have not been observed previously. Another advantage of using in situ observation in this study is shown to provide direct evidence of the loop-loop structure transformation. With the establishment of a theoretical framework of double-layer loop formation energy via molecular dynamics (MD) computer simulations, our study could provide fundamental understanding of the formation of the c-component dislocation loops with multiple-layer structures and potentially help advance the theoretical modeling in developing future nuclear materials with high irradiation resistance.

\section{Experimental Procedures}

\section{$\underline{2.1 \text { Sample preparation }}$}

Commercial purity magnesium (99.9\%) was used for this study. The major impurity elements in this composition are $0.0510 \% \mathrm{Fe}, 0.0320 \% \mathrm{Mn}, 0.0089 \% \mathrm{C}, 0.0054 \% \mathrm{Al}, 0.0027 \% \mathrm{Na}$, $0.0026 \% \mathrm{Zn}, 0.0010 \% \mathrm{H}$, and $0.0007 \% \mathrm{O}$ in weight percent. TEM sample was prepared by the electron-polishing method using a Struers TenuPol-2 electro-polishing machine under $-30{ }^{\circ} \mathrm{C}$ and 
$200 \mathrm{~mA}$ current. The electrolyte contains $5.3 \mathrm{~g}$ lithium chloride, $11.16 \mathrm{~g}$ magnesium perchlorate, $100 \mathrm{ml}$ 2-butoxy-ethanol and $500 \mathrm{ml}$ methanol. Then, the TEM sample is low energy ion-milled on a cold stage and plasma cleaned for HRTEM and STEM observation.

\section{$\underline{2.2 \text { TEM and STEM Observations }}$}

Electron irradiations and in situ dynamic observations were operated simultaneously inside a

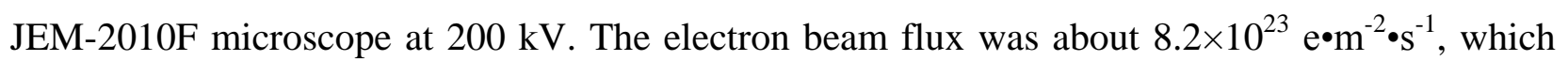
corresponds to a damage rate of $1.4 \times 10^{-3} \mathrm{dpa} \mathrm{s}^{-1}$. To determine the dislocation loop structure in the STEM mode, the specimen was in situ irradiated in an FEI Titan G2 microscope in the TEM mode operated at $200 \mathrm{kV}$, with a beam flux of $6.3 \times 10^{22} \mathrm{e} \cdot \mathrm{m}^{-2} \cdot \mathrm{s}^{-1}$ dpa (a damage rate of $1.1 \times 10^{-4}$ $\mathrm{dpa} \cdot \mathrm{s}^{-1}$ for 1 hour). Afterward, the microscope was switched to STEM mode for atomic resolution HAADF imaging. The probe was aberration-corrected with a probe size of about $0.08 \mathrm{~nm}$. The probe current, convergence angle, and collection inner semi-angle were $30 \mathrm{pA}, 21 \mathrm{mrad}$ and 77 mrad, respectively. The atomic column in Z-contrast imaging in HAADF-STEM directly corresponds to the real atomic column position in the crystal, which allows faithful interpretation of the atomic stacking sequence and dislocation structure of multiple-layer loops in hcp $\mathrm{Mg}$.

\section{$\underline{\text { 2.3 Molecular Dynamics Simulations }}$}

Molecular dynamics simulations were conducted using the Large-scale Atomic/Molecular Massively Parallel Simulator (LAMMPS) [40]. Formation energies of single- and double-layer loops were calculated for different configurations identified in the experiment. The specific formation energies of the second loop of double-layer loops were derived from the results. The effect of strain/stress on the formation energy was also studied using the selected loops. Specifically, loops were constructed in an approximated circular shape containing 100 to 8000 
SIAs. In total, the simulation cells contained about 4.6 M atoms. Such big cells were found sufficient to eliminate the size effect as big loops were considered. Two types of single-layer loops were calculated, i.e., E and I1. For double-layer loops, the first loop is constructed in either E or I1 type containing 6051 SIAs. The diameter of such loop is about $26 \mathrm{~nm}$, which is close to the observed critical size of double-layer loop formation in the experiment. The second loop was further built according to the structure determined by the experiment in this study. We varied the size of the loop from about 100-1900 SIAs for the second loop. Four types of double-layer loops were compared in this study (will be defined in a later section) and being relaxed for the energy calculation. To obtain the formation energies, the simulation cells were relaxed at $0 \mathrm{~K}$ until the potential energy converged. The interatomic interaction was described by the embedded-atom-method (EAM) potential developed by Liu at al. [41]. This potential accurately reproduces the cohesive energy $(1.51 \mathrm{eV})$ and the $\mathrm{I} 2$ stacking fault formation energy $\left(54.5 \mathrm{~mJ} / \mathrm{m}^{2}\right)$ of hcp $\mathrm{Mg}$ versus those measured in experiments, $1.51 \mathrm{eV}$ and $60 \mathrm{~mJ} / \mathrm{m}^{2}$, respectively. Therefore, it is suitable for this study. The formation energy normalized to per SIA, $E^{f}$, is given by:

$$
E^{f}\left(N^{S I A}\right)=\left(E-E_{0}\right) / N^{S I A}
$$

Here, $E$ is the energy of a cell containing $N^{S I A}$ SIAs in the desired configuration, and $E_{0}$ the total energy of a defect free cell with the same number of atoms. The specific formation energy of the second loop, $E_{2}^{f}$, is defined using a reference cell containing 6051 SIAs in a single-layer loop of either E or I1 configuration, with a total energy of $E_{0}+6051 * E^{f}(6051)$, by:

$$
E_{2}^{f}=\left(E-E_{0}-6051 * E^{f}(6051)\right) / N_{2}^{S I A}
$$


Here $E$ is the energy of a cell containing a double-layer loop with $N^{S I A}$ SIAs, and $E_{0}$ the total energy of a defect free cell with the same number of atoms. $E_{2}^{f}$ is normalized using the number of SIAs in the second loop, $N_{2}^{S I A}$, and the total SIA number contained is $N^{S I A}=6051+N_{2}^{S I A}$.

\section{Results and Discussion}

\section{$\underline{3.1 \text { In situ Observation of Double-layer Loop Formation and Growth }}$}

The complete formation process of a double-layer c-component dislocation loop in $\mathrm{Mg}$ was observed under in situ HRTEM (see supplementary video 1). A series of time-resolved images from the video are shown in Figure 1. At the beginning, a single-layer SIA dislocation loop was first nucleated and grew on (0001) basal plane projected in a line-shaped morphology in Figure 1a. After the single-layer loop extended to $25-100 \mathrm{~nm}$, a contrast change was noticed in HRTEM image close to the center region of the first faulted loop (pointed out by an arrow in Figure 1a). Such a contrast change is attributed to the arising of local lattice strain, indicating the nucleation of a new interstitial layer on the basal plane, i.e., a second loop. The initial growth rate of double-layer loops is about $0.2-0.35 \mathrm{~nm} / \mathrm{s}$ in diameter for the first layer loop, and about 0.07-0.25 $\mathrm{nm} / \mathrm{s}$ in diameter for the second layer loop according to HRTEM observation. The interstitial nature of the second loop is further confirmed when it grew into a larger size, whose $1 / 2<0001>$ Burgers vector component can be identified from HRTEM imaging as pointed out by the arrows in Figure 1c. Meanwhile, the first loop continued to grow on the (0001) basal plane until it started to interact with neighboring loops, as seen in Figure 2. The double-layer loop could grow to a size of 50-200 nm, as revealed by dislocation contrast from weak beam dark-field (WBDF) TEM imaging in the irradiated area. The WBDF image also showed dark contrast inside the second 
loop, which is different from the brighter contrast in between the first and second loop (dashed region in Figure 1d), suggesting the removal of stacking fault inside the second loop.

Loop-loop interaction was observed when the edges of neighbor loops were extending toward each other. Figure 2 shows a typical case in which three loops involved in (see more details in supplementary video 2). The top and bottom loops were growing and extending to the left side of the figure, while the middle one was growing and extending to the right. The c-component dislocation loop usually interacts with each other in a head-on-head way. As outlined by dashed ellipses in Figure $2 \mathrm{~b}$ and $\mathrm{d}$, the loop edge (as pointed out by arrow) extended until it was lined up with other loop edges along <0001> direction. Such manner is also observed from a WBDF imaging in Figure 1d as pointed out by the arrows. Note that the loop growth rate will decrease significantly when the loop edges met and aligned head-on-head. The loop-loop interaction could be strong enough to stop or even push back the loop extension as illustrated in the dynamic process between the middle and the bottom side of the loop in Figure 2b-d and supplementary video 2. As a result, network of these c-component dislocation loops can be formed (Figure 1d) when the loop extension is suppressed. Such network structure provides a relatively stable environment for further void growth in the later stage as revealed in [35].

\section{$\underline{3.2 \text { Versatile Structure of the Double-layer Loops }}$}

It is found that c-component double-layer loops can form diverse configurations in hcp $\mathrm{Mg}$. Depending on its first and second loops types, Figure 3 summarized all four possible double-layer loop structures that were observed, named as (a) E-N type, (b) I1-I2 type, (c) I1-N $\mathrm{N}_{\mathrm{a}}$ type, and (d) I1- $\mathrm{N}_{\mathrm{b}}$ type. The detailed atomic structure of these four types of double-layer loops are presented in Figure 4, and the corresponding formation path of double-layer loops from single-layer E or I1 
loops, and their possible transformation are also proposed in the figure. For the convenience of discussion, we name the double-layer loop structures based on their stacking-fault types. Take the E-N type of double-layer loops for example, E denotes extrinsic-type of stacking fault for the first loop; $\mathrm{N}$ represents the perfect crystal restored by the second loop. In most cases, the stacking fault configuration is unique to the specific type of the double-layer loop structure, providing us a convenient way to distinguish the loop structure by identifying the stacking fault structure from atomic HAADF-STEM images.

According to the in situ observation in Figure 1, the formation of double-layer loop appears to be a two-step process via forming a precursor loop at beginning, and then nucleating the second loop next. For the E-N type double-layer loop shown in Figure 3a, its first loop has a Burgers vector of $\mathbf{b}_{1}=1 / 2[0001]$, and contains an extrinsic (E) type stacking fault with $A B A B C A B A B$ stacking sequence. The underline label $\underline{\mathrm{BCA}}$ indicates the stacking sequences to be different from the ideal $A B A B A B A B$ stacking in hcp crystal (labeled as $\mathrm{N}$ ). The second layer of interstitial atoms could further results the stacking sequences as $\mathrm{ABA} \underline{\mathrm{BA} * \mathrm{CAB}} \mathrm{AB}$ or $\mathrm{AB} \underline{\mathrm{ABCB} * \mathrm{ABAB}(*}$ indicates the add-on second interstitial layer to the first sequence), and the perfect staking sequence can be restored by locally shuffling the neighboring interstitial atom from $\mathrm{C}$ to $\mathrm{B}$ position (or C to A). Thus, a second interstitial loop with a Burgers vector of $\mathbf{b}_{2}=1 / 2[0001]$ is formed. The corresponding reaction can be expressed as:

$$
b_{E N}=\frac{1}{2}[0001]+\frac{1}{2}[0001]=[0001]
$$


Figure 4 (mid-left) shows the experimental observation of E-N double-layer loop configuration. The c-component and its stacking fault sequence of the first and second loops are illustrated in the corresponding schematic drawing.

For the I1-I2 type loop shown in Figure $3 \mathrm{~b}$ and Figure 4 (mid-right), the first loop has a Burgers vector of $\mathbf{b}_{1}=1 / 6[2 \overline{2} 03]$ and an intrinsic (I1) type stacking fault with $\mathrm{ABA} \underline{\mathrm{B} C B C B}$ stacking sequence. I1 faulted loop could be directly transferred from E type of single-layer loop via $1 / 3[1 \overline{1} 00]$ partial dislocation shearing. The shear is suggested via the partial dislocation nucleation and extension process on the loop plane as revealed in fcc crystal from MD simulation $[42,43]$. Such I1 stacking fault also allows the formation of an extra SIA layer on the first one, i.e., a second loop with Burgers vector $\mathbf{b}_{2}=1 / 2[0001]$. Therefore, the total Burgers vector of the double-layer loops would be:

$$
b_{I 1 I 2}=\frac{1}{6}[2 \overline{2} 03]+\frac{1}{2}[0001]=\frac{1}{3}[1 \overline{1} 03]
$$

Unlike the E-N type, the second loop does not erase the stacking fault from the first loop; rather it transforms the I1 stacking fault into a higher energy stacking fault configuration of I2 type (ABABACBCB sequence). This double-layer loop with high stacking fault energy is for the first time to be experimentally observed in hcp structures. The atomic configuration is shown in Figure 4 (mid-right).

The I1-I2 type loops can further lower the energy from an unfaulted process via a dislocation reaction with a Shockley partial loop shear on the stacking fault plane as:

$$
\frac{1}{3}[1 \overline{1} 03]+\frac{1}{3}[\overline{1} 100] \rightarrow[0001]
$$


It forms an I1-N $\mathrm{N}_{\mathrm{a}}$ type of double-layer loop with $\mathbf{b}_{\mathbf{1}}=1 / 6[2 \overline{2} 03], \mathbf{b}_{\mathbf{2}}=1 / 6[\overline{2} 203]$, and $\mathbf{b}_{\mathbf{I 1}-\mathrm{Na}}=[0001]$. Figure 5 shows the experimental evidence of such transformation captured by in situ STEM observations. The intermediate process of $\mathrm{I} 1-\mathrm{N}_{\mathrm{a}}$ formation through Eqs. (4) and (5) are thus suggested as in dislocation reaction in Eq. (6) [31, 44]. This bridges the conventional understanding of I1-N type of double-layer loop formation in hcp Zn [44] and Zr [31].

$$
\frac{1}{6}[2 \overline{2} 03]+\frac{1}{6}[\overline{2} 203] \rightarrow[0001]
$$

The structure of the I1- $\mathrm{N}_{\mathrm{a}}$ type double-layer loop is shown in Figure 4c (bottom-right). Note that the I1- $\mathrm{N}_{\mathrm{a}}$ double-layer loop is also possible to be transformed from an E-N single-layer loop via a similar Shockley partial dislocation shear on the residual faulted plane between the first and second loop; however, such transformation is not observed in this study.

In a similar manner, the unfaulted process can occur via the following dislocation reaction

$$
\frac{1}{3}[1 \overline{1} 03]+\frac{1}{3}[0 \overline{1} 10] \rightarrow \frac{1}{3}[1 \overline{2} 13]
$$

An I1- $\mathrm{N}_{\mathrm{b}}$ type of double-layer loop is thus formed with $\mathbf{b}_{\mathbf{1}}=1 / 6[2 \overline{2} 03], \mathbf{b}_{\mathbf{2}}=1 / 6[0 \overline{2} 23]$, and $\mathbf{b}_{\mathbf{I 1}-\mathbf{N b}}=$ $1 / 3[1 \overline{2} 13]$. This $\mathrm{I} 1-\mathrm{N}_{\mathrm{b}}$ type double-layer loop has the same stacking sequences on the first and second loop as those of $\mathrm{I} 1-\mathrm{N}_{\mathrm{a}}$, but contains a full dislocation component of $<\mathrm{a}>$. The I1- $\mathrm{N}_{\mathrm{b}}$ type double-layer loop can be distinguished from I1- $\mathrm{N}_{\mathrm{b}}$ type via the Burgers circuit as compared in Figure 4 (bottom-right and bottom-left).

Figure 6 shows the relative fraction of the four types of double-layer loops observed in the irradiated area. Their abundance is estimated from 47 double-layer loops observed in the STEM. Among them, I1- $\mathrm{N}_{\mathrm{a}}$ type is the most frequently observed and maintains a relative fraction of 
about $69 \%$. The E-N type and I1-I2 type loops constitute 14\% and 15\%, respectively. Interestingly, a small number of E-N type and I1-I2 type loops are still detected. The existence of I1-I2 type double-layer loop indicates that I1-I2 type loop may still maintain the stability to prevent them from transforming into the I1- $\mathrm{N}_{\mathrm{a}}$ type. Although I1- $\mathrm{N}_{\mathrm{b}}$ type double-layer loop has been reported [45], such double-layer loop type is not commonly seen from our current study as compared with other three types of double-layer loops.

To understand the observed diverse c-component dislocation loop configurations, the formation energies of all loop configurations were calculated via molecular dynamics (MD) simulations and are compared in Figure 7. In the calculation, the size of the first loop was fixed at about $26 \mathrm{~nm}$ (i.e., with 6051 SIAs), which is comparable to the observed critical size for a second loop to nucleate and grow. As the second loop becomes larger to about 1700 SIAs, a trend regarding the formation energy of double-layer loops is clearly seen from Figure $7 \mathrm{~b}$ as I1- $\mathrm{N}_{\mathrm{a}}<$ E-N $<$ I1-I2 $<$ I1- $\mathrm{N}_{\mathrm{b}}$. This explains why the I1- $\mathrm{N}_{\mathrm{a}}$ double-layer loop is the most commonly observed loop type.

The above ranking of loop energy, however, is not apparent at the beginning stage of a double-layer loop formation. Very similar formation energies are found between I1- $\mathrm{N}_{\mathrm{a}}$ and I1-I2 loop, when the second loop is within $8 \mathrm{~nm}$ size (i.e., less than 600 SIAs). This indicates that the formation of I1-I2 loop could also be favorable at the beginning. In fact, I1-I2 loop has smaller dislocation line energy $\left(\mathbf{b}_{2}=1 / 2[0001]\right)$ than that of I1-N loop $\left(\mathbf{b}_{2}=1 / 6[\overline{2} 203]\right)$, which compensates its high 12 stacking fault energy in a small loop size. Their comparable formation energy also suggests that the I1-I2 double-layer loop may not necessarily transform to I1-N loop right after its formation. In addition, the structural transformation from I1-I2 to I1-N type of loop requires a 
$1 / 3[1 \overline{1} 00]$ partial dislocation nucleation and propagation on the faulted loop plane, which further increases the energy barrier to stabilize the I1-I2 loop in its larger size. As a consequence, I1-I2 loop can be retained in the microstructure as evidenced from the experiment. When locally energized by the high intensity electron beam during STEM imaging, such I1-I2 double-layer loop in a metastable state will eventually transfer to a lower energy I1-N loop.

The E-N type double-layer loops appear to have much higher formation energy than the other types in their early stage of formation (as shown in Figure 7b). However, such high double-layer loop-layer energy is due to the significant contribution from its first E-type faulted loop, whose formation energy is larger than I1-type. For a faithful comparison, we further calculated the specific formation energy of the second loop without including the first loop. As seen in Figure 8, the second loop in E-N type has the lowest specific formation energy, which suggests a large likehood/tendency to form a second layer on a single-layer E-type loop. Thus, the formation of E-N loop is possible even with its high formation energy at the beginning.

Here, it should be noted that the existence of the E-N type double-layer loop is dependent on the stability of pre-existing single-layer E-type loop in microstructure. This prerequisite can be affirmed via the calculation of single-layer E and I1 type of loop formation energy as shown in Figure 7 and 8. A crossover of the formation energy in E-type and I1-type single-layer loops is seen in Figure 7a with the loop size less than $11 \mathrm{~nm}$ (1000 SIAs), implying that the E-type loops can be favorably formed in a smaller loop size. In fact, E-type loops may still co-exist with I1-type in the microstructure in a relatively larger loop size due to the difficulty of partial nucleation for the E-to-I1 loop transformation. As E-N double-layer loop is formed, such structural transformation becomes even more difficult since the faulted loop area is squeezed as 
the second loop edge extends much closer to the first loop edge in less than $5 \mathrm{~nm}$ (as shown earlier in Figure 4a). No experimental evidence for the direct transformation of E-N loop to I1-N type has been observed, which further confirms the stability of E-N type loop.

\subsection{Multi-layer dislocation loops}

Although the stacking fault energy of a single-layer loop can be reduced via the growth of the second loop on its faulted plane, it should not be the only driving force for the double-layer loop formation. As shown in the calculation in Fig. 7, all types of double-layer loops experience an increment in their normalized formation energy during their nucleation. Such an increment is largely attributed to the normalized dislocation line energy of the second loop, i.e., there exists an energy barrier for the second loop nucleation. For I1-I2 double-layer loop, the second loop formation even increases the loop stacking fault energy in additional to the dislocation line energy. To overcome the energy barrier, the critical role of high energy state of SIA sink to the double-layer loop is suggested. As known, Frankel pairs of SIA and vacancy can be generated during electron irradiation. Those uncombined SIAs have much higher formation energy of about $1.51 \mathrm{eV}$ calculated from MD, which will further agglomerate in the form of SIAs loops to reduce the excess energy. The large energy drop from $1.51 \mathrm{eV}$ to $0.45-0.2 \mathrm{eV}$ could promote both singleand double-layer loop formation as seen from Figure 8. A competitive process for loop formation between a new single-layer loop and a double-layer loop would therefore be expected. In fact, as the loops formed networks during the irradiation, we observed an average loop spacing of about 10-15 $\mathrm{nm}$ when loops appear to saturate in the microstructure. The high density of loops further reduced the possibility of nucleating new single-layer loops, while providing alternative sites for SIA to nucleate and form the new layer on top of the existing loops. 
As SIAs were continuously generated and agglomerated during irradiation, the formation of triple or even quadruple loops is expected. Indeed, I1-I2-I1 type and I1-N-I1 type of triple loops are observed and shown in Figure 9. Quadruple multiple layer loops are also observed in types of I1-N-I1-I2 and I1-I2-I1-N. Table 1 summarizes the predicted type of double-, triple- and quadruple-layer loops and their Burgers vector. The observed types of multiple-layer loops are also marked.

\section{$\underline{3.5 \text { Effect on loop-loop interaction }}$}

The formation of c-component interstitial loops can cause compressive strain along [0001] direction. As shown in Figure 10, the compressive/tensile strain is applied along [0001] direction for up to $2 \%$ in single- and double-layer loops. In general, for all loops, the formation energy increases (decreases) upon compressive (tensile) strain. As two loops approach each other, their stress fields overlap and interact. Specifically, as the tensile stress is generated by one loop near its outer area of loop edge, the reduced formation energy promotes the growth of another loop until the two loop edges are aligned (as seen in Figure 2c). Thus, two loops tend to be attracted to each other and line up in head-on-head configuration. However, as one loop further extends to the inner side of another loop, the stress condition is reversed to a compression stress to suppress the loop extension further. It should also be noted that the formation energy of loop is only slightly dependent on the strain shown in Figure 10. The small amount of increased loop energy barrier for loop extension may be easily overcome by the by absorbing isolated SIAs to a loop, thus initiating further passing the head-on-head loop position and further growth (as seen in Figure $2 d)$. 


\section{Conclusion}

In summary, we have directly observed in situ the interstitial c-component dislocation loops in $\mathrm{Mg}$ under electron irradiation. The detailed atomic structure of loops and their associated stacking faults were characterized via STEM HAADF imaging. These c-component dislocation loops are frequently observed in double-layer loop structures. Inter-loop interaction occurred in forms of double-layer loop formation, extended single-layer loop formation, and head-on-head configuration. Four types of double-layer loops were observed, which are categorized as (a) E-N, (b) I1-I2, (c) I1- $\mathrm{N}_{\mathrm{a}}$, and (d) $\mathrm{I} 1-\mathrm{N}_{\mathrm{b}}$. Their relative fractions are $14 \%, 15 \%, 69 \%$ and $2 \%$, respectively. The evidence of loop-to-loop transformation was in situ captured in this study. Structural transformation occurred through a partial dislocation shearing or un-faulted process.

Based on molecular dynamics calculations, the formation energies and relative stabilities of the four types of double-layer loops can be ranked as I1- $\mathrm{N}_{\mathrm{a}}<\mathrm{E}-\mathrm{N}<\mathrm{I} 1-\mathrm{I} 2<\mathrm{I} 1-\mathrm{N}_{\mathrm{b}}$, consistent with their abundance in observations. At the initial stage of their formation, however, energy calculation shows the possibility of forming I1-I2 and E-N type of loops, whose metastable structure could be retained in the microstructure. We believe that the formation of double-layer loops could be related to the self-interstitial atoms whose agglomeration into c-component dislocation loops could effectively reduce the system energy. The c-component dislocation loops provide new sites for SIA sink to form multiple-layer loops, which compete with the nucleation of new single-layer loops in the matrix. This promoted the formation of triple and quadruple c-component dislocation loops with diverse structures. 


\section{Acknowledgement}

We acknowledge financial support from the Laboratory Directed Research and Development Program Office of the Idaho National Lab oratory (00042959-00032), the Department of Energy Idaho Field Office (DE-NE0000538), and U.S. Army Research Office (W911NF-12-1-0009). EJL would like to acknowledge NSF-CMMI-1631873, for support of his research. This work was performed in part at the Analytical Instrumentation Facility (AIF) at North Carolina State University, which is supported by the State of North Carolina and the National Science Foundation (award number ECCS-1542015). The AIF is a member of the North Carolina Research Triangle Nanotechnology Network (RTNN), a site in the National Nanotechnology Coordinated Infrastructure (NNCI).

\section{Reference}

[1] B.N. Singh, S.I. Golubov, H. Trinkaus, D.J. Edwards, M. Eldrup, Review: Evolution of stacking fault tetrahedra and its role in defect accumulation under cascade damage conditions, J Nucl Mater 328(2-3) (2004) 77-87.

[2] C.H. Woo, Defect accumulation behaviour in hcp metals and alloys, J Nucl Mater 276(1-3) (2000) 90-103.

[3] Y.N. Osetsky, M. Victoria, A. Serra, S.I. Golubov, V. Priego, Computer simulation of vacancy and interstitial clusters in bcc and fcc metals, J Nucl Mater 251 (1997) 34-48.

[4] D.I.R. Norris, Voids in irradiated metals (Part I), Radiation Effects 14(1-2) (1972) 1-37.

[5] M. Griffiths, Evolution of Microstructure in Hcp Metals during Irradiation, J Nucl Mater 205 (1993) 225-241.

[6] F.A. Garner, Evolution of microstructure in face-centered cubic metals during irradiation, J Nucl Mater 205 (1993) 98-117.

[7] W. Xu, L. Li, J.A. Valdez, M. Saber, Y. Zhu, C.C. Koch, R.O. Scattergood, Effect of nano-oxide particle size on radiation resistance of iron-chromium alloys, J Nucl Mater 469 (2016) 72-81.

[8] M. Kiritani, Defect interaction processes controlling the accumulation of defects produced by high energy recoils, J Nucl Mater 251 (1997) 237-251.

[9] Y. Zhang, H. Huang, P.C. Millett, M. Tonks, D. Wolf, S.R. Phillpot, Atomistic study of grain boundary sink strength under prolonged electron irradiation, J Nucl Mater 422(1-3) (2012) 69-76. 
[10] S.J. Zinkle, P.J. Maziasz, R.E. Stoller, Dose dependence of the microstructural evolution in neutron-irradiated austenitic stainless steel, J Nucl Mater 206(2-3) (1993) 266-286.

[11] M. Griffiths, Microstructure Evolution in Hcp Metals during Irradiation, Philos Mag A 63(5) (1991) 835-847.

[12] M. Kiritani, Microstructure evolution during irradiation, J Nucl Mater 216 (1994) 220-264.

[13] C.H. Woo, B.N. Singh, A.A. Semenov, Recent advances in the understanding of damage production and its consequences on void swelling, irradiation creep and growth, J Nucl Mater 239 (1996) 7-23.

[14] D. Kulikov, M. Hou, Vacancy dislocation loops in zirconium and their interaction with self-interstitial atoms, J Nucl Mater 342(1-3) (2005) 131-140.

[15] Y.N. Osetsky, D.J. Bacon, F. Gao, A. Serra, B.N. Singh, Study of loop-loop and loop-edge dislocation interactions in bcc iron, J Nucl Mater 283-287, Part 2 (2000) 784-788.

[16] Z. Yang, N. Sakaguchi, S. Watanabe, M. Kawai, Dislocation Loop Formation and Growth under In Situ Laser and/or Electron Irradiation, Sci. Rep. 1 (2011) 190.

[17] A.K. Khan, Z. Yao, M.R. Daymond, R.A. Holt, Effect of foil orientation on damage accumulation during irradiation in magnesium and annealing response of dislocation loops, J Nucl Mater 423(1-3) (2012) 132-141.

[18] F. Wan, Q. Zhan, Y. Long, S. Yang, G. Zhang, Y. Du, Z. Jiao, S. Ohnuki, The behavior of vacancy-type dislocation loops under electron irradiation in iron, J Nucl Mater 455(1-3) (2014) 253-257.

[19] D. Terentyev, L. Malerba, Interaction of a screw dislocation with Cu-precipitates, nanovoids and Cu-vacancy clusters in BCC iron, J Nucl Mater 421(1-3) (2012) 32-38.

[20] M. Wen, A. Takahashi, N.M. Ghoniem, Kinetics of self-interstitial cluster aggregation near dislocations and their influence on hardening, J Nucl Mater 392(3) (2009) 386-395.

[21] A. Berghezan, A. Fourdeux, S. Amelinckx, Transmission electron microscopy studies of dislocations and stacking faults in a hexagonal metal: Zinc, Acta Metallurgica 9(5) (1961) 464-490.

[22] Y. Hamaguchi, H. Kuwano, T. Misawa, R. Miura, T. Yamada, The $11 \mathrm{MeV}$ proton irradiation behavior of ferritic steels, J Nucl Mater 141-143, Part 2 (1986) 781-785.

[23] C.H. Woo, X. Liu, Generation of c-component edge dislocations in $\alpha$-zirconium during neutron irradiation - An atomistic study, J Nucl Mater 393(3) (2009) 513-517.

[24] L. Tournadre, F. Onimus, J.L. Béchade, D. Gilbon, J.M. Cloué, J.P. Mardon, X. Feaugas, O. Toader, C. Bachelet, Experimental study of the nucleation and growth of c-component loops under charged particle irradiations of recrystallized Zircaloy-4, J Nucl Mater 425(1-3) (2012) 76-82.

[25] S. Yamada, T. Kameyama, Observation of c-component dislocation structures formed in pure $\mathrm{Zr}$ and Zr-base alloy by self-ion accelerator irradiation, J Nucl Mater 422(1-3) (2012) 167-172.

[26] N. Gharbi, F. Onimus, D. Gilbon, J.P. Mardon, X. Feaugas, Impact of an applied stress on c-component loops under Zr ion irradiation in recrystallized Zircaloy-4 and M5, J Nucl Mater 467, Part 2 (2015) 785-801.

[27] C. Dai, L. Balogh, Z. Yao, M.R. Daymond, Atomistic simulations of the formation of <c>-component dislocation loops in $\alpha$-zirconium, J Nucl Mater 478 (2016) 125-134.

[28] A. Jostsons, R.G. Blake, J.G. Napier, P.M. Kelly, K. Farrell, Faulted loops in neutron-irradiated zirconium, J Nucl Mater 68(3) (1977) 267-276.

[29] G.J.C. Carpenter, R.H. Zee, A. Rogerson, Irradiation growth of zirconium single crystals: A review, J Nucl Mater 159 (1988) 86-100.

[30] M. Griffiths, A review of microstructure evolution in zirconium alloys during irradiation, J Nucl Mater 159 (1988) 190-218. 
[31] M. Griffiths, R.W. Gilbert, The formation of c-component defects in zirconium alloys during neutron irradiation, J Nucl Mater 150(2) (1987) 169-181.

[32] D. Faulkner, C.H. Woo, Void swelling in zirconium, J Nucl Mater 90(1-3) (1980) 307-316.

[33] M. Griffiths, C.D. Cann, R.C. Styles, Neutron irradiation damage in $64 \%$ cold-worked Titanium, Journal of Nuclear Materials 149(2) (1987) 200-211.

[34] W. Xu, Y. Zhang, G. Cheng, W. Jian, P.C. Millett, C.C. Koch, S.N. Mathaudhu, Y. Zhu, In-situ atomic-scale observation of irradiation-induced void formation, Nat Commun 4 (2013) 2288.

[35] W.Z. Xu, Y.F. Zhang, G.M. Cheng, W.W. Jian, P.C. Millett, C.C. Koch, S.N. Mathaudhu, Y.T. Zhu, Dynamic Void Growth and Shrinkage in Mg under Electron Irradiation, Materials Research Letters 2(3) (2014) 1-8.

[36] M.E. Whttehead, A.S.A. Karim, M.H. Loretto, R.E. Smallman, Electron radiation damage in H.C.P. metals-II. The nature of the defect clusters in $\mathrm{Zn}$ and $\mathrm{Cd}$ formed by irradiation in the HVEM, Acta Metallurgica 26(6) (1978) 983-993.

[37] J.S. Lally, P.G. Partridge, Observations in quenched magnesium, Philosophical Magazine 13(121) (1966) 9-30.

[38] R. Hales, R.E. Smallman, P.S. Dobson, The Formation and Growth of Vacancy Loops in Magnesium, Proceedings of the Royal Society of London. Series A. Mathematical and Physical Sciences 307(1488) (1968) 71-81.

[39] D.J. Bacon, R. Bullough, The nucleation and growth of multi-layer defects in quenched aluminium, Philosophical Magazine 18(153) (1968) 561-571.

[40] S. Plimpton, Fast Parallel Algorithms for Short-Range Molecular Dynamics, J. Comput. Phys. 117(1) (1995) 1-19.

[41] L. Xiang-Yang, B.A. James, E. Furio, A.M. John, EAM potential for magnesium from quantum mechanical forces, Modelling and Simulation in Materials Science and Engineering 4(3) (1996) 293.

[42] T. Kadoyoshi, H. Kaburaki, F. Shimizu, H. Kimizuka, S. Jitsukawa, J. Li, Molecular dynamics study on the formation of stacking fault tetrahedra and unfaulting of Frank loops in fcc metals, Acta Mater 55(9) (2007) 3073-3080.

[43] W. Zhu, W. Yang, Molecular dynamics study of configuration and stability of vacancy clusters in fcc Ag, Philosophical Magazine 91(29) (2011) 3793-3809.

[44] M.E. Whitehead, A.S.A. Karim, M.H. Loretto, R.E. Smallman, Electron radiation damage in H.C.P. metals-II. The nature of the defect clusters in $\mathrm{Zn}$ and $\mathrm{Cd}$ formed by irradiation in the HVEM, Acta Metallurgica 26(6) (1978) 983-993.

[45] A.K. Khan, Z. Yao, M.R. Daymond, Microstructure evolution during electron and ion irradiation in commercial purity magnesium, Philosophical Magazine 94(17) (2014) 1-15. 


\section{Figures and Captions}
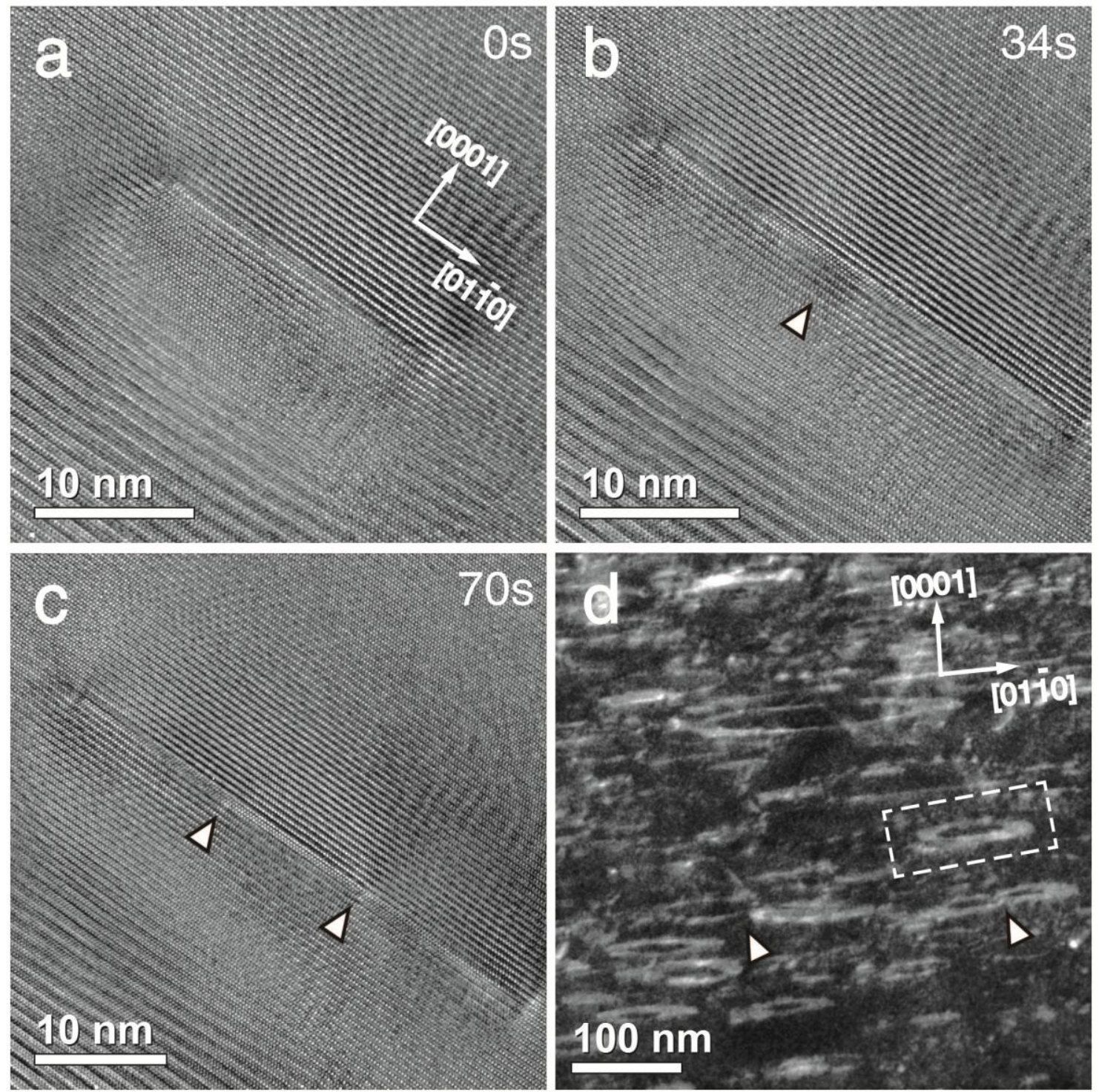

Figure 1. In situ observation of a double-layer loop formation under electron radiation (see also supplementary video 1). a, A single-layer interstitial c-component dislocation loop is growing along (0001) basal plane (0.03 dpa). b, A second interstitial layer is nucleated on top of the first loop as distinguished from the strain contrast pointed out by the arrow (0.08 dpa). c, The second loop grows larger after nucleation $(0.13 \mathrm{dpa})$. d, Low magnification TEM image showing the loop network in the sample via weak-beam dark-field imaging. One double-layer loop is highlighted by the dashed line, and a head-on-head interaction between two loops are pointed by arrows. 


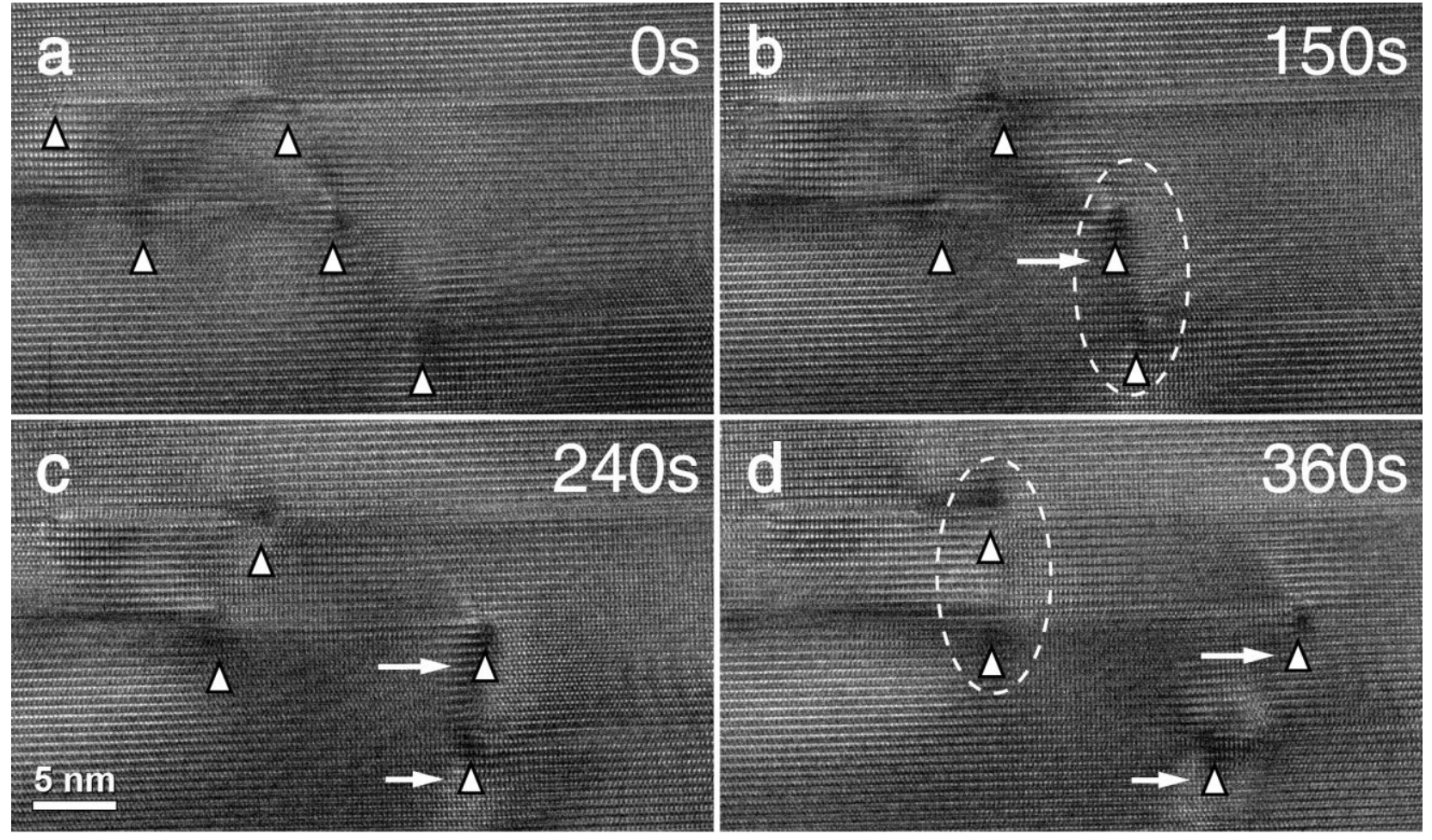

Figure 2. A series of HRTEM images showing loop-loop interactions in hcp Mg under e-beam irradiation. a, Two double-layer loops are seen in the top and middle area of the image, whose loop edges are pointed out by the arrows. $b$, The edge of the middle loop extends to the right side of the image until it is lined up with the single-layer loop at the bottom side. Two loops are aligned up in a head-on-head position as marked by the dashed circle (c), until the middle loop further extends and crosses over (d). 
a

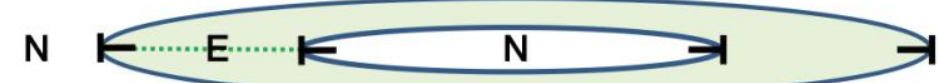

$$
b_{1}=\frac{1}{2}[0001] \quad b_{2}=\frac{1}{2}[0001] \quad b_{1}+b_{2}=[0001]
$$

b

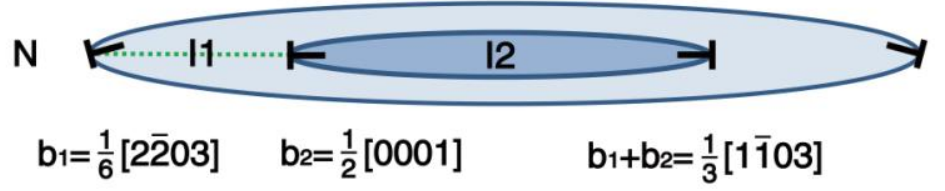

C
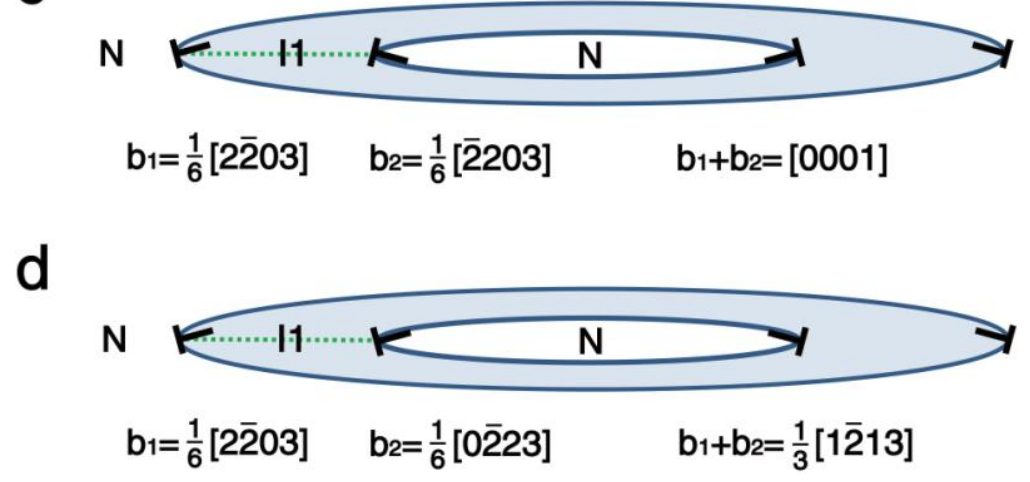

Figure 3. Illustration of loop configuration of possible double-layer loops in a), E-N, b), I1-I2, c), I1- $\mathrm{N}_{\mathrm{a}}$ and d), I1- $\mathrm{N}_{\mathrm{b}}$ types. Loop areas in green, blue and dark blue colors represent the stacking fault with E, I1, and I2 type, respectively. The area containing no stacking faults is marked as N. The corresponding Burgers vector of the first loop, second loop, and their summation are also informed. 


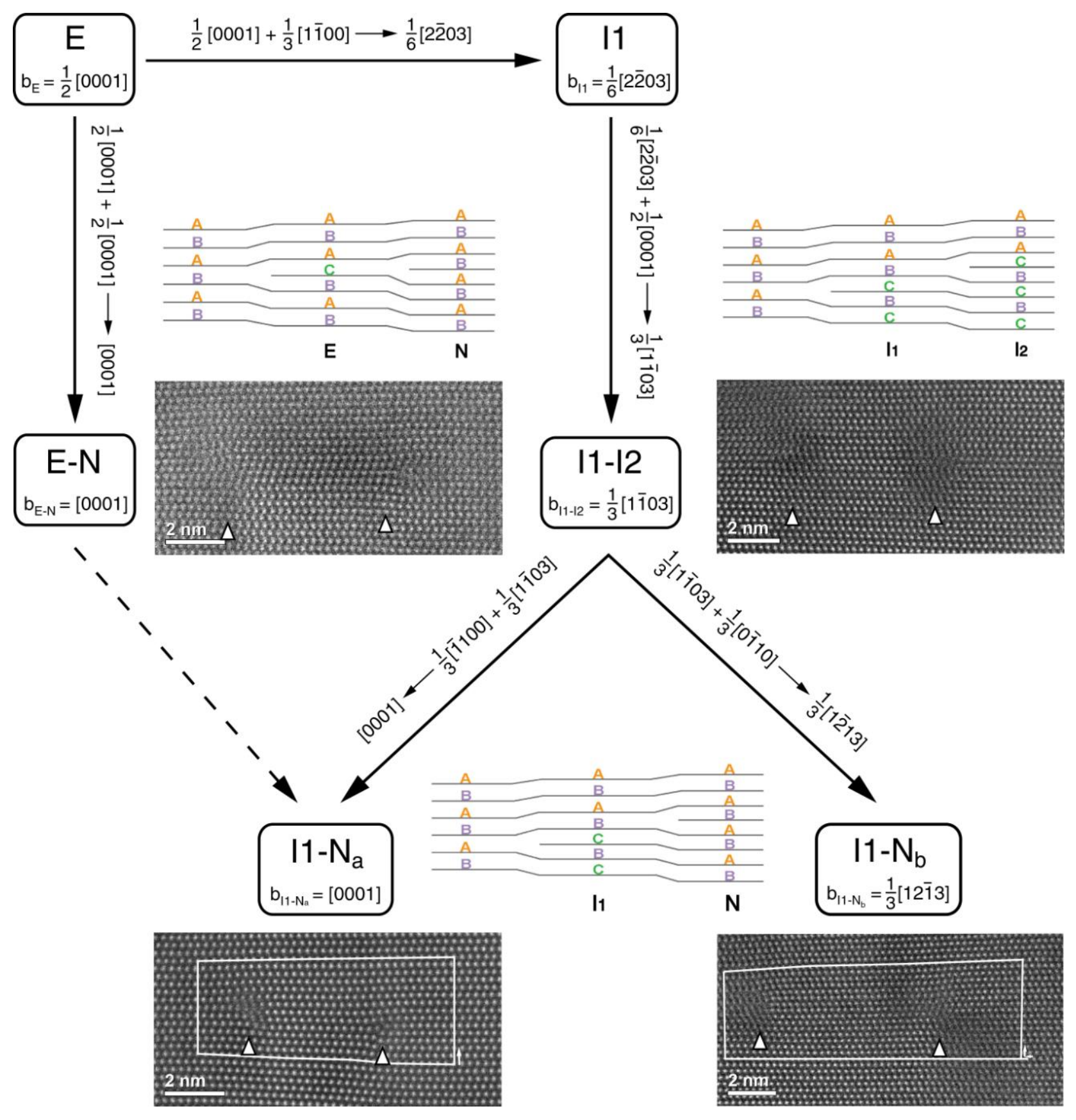

Figure 4. Chart of c-component dislocation formation sequences and atomic structure of double-layer loop series of E-N, I1-I2, I1- $\mathrm{N}_{\mathrm{a}}$ and I1- $\mathrm{N}_{\mathrm{b}}$ types. Interstitial layers and stacking fault configuration of the corresponding double-layer loops are illustrated for comparison. Additional $<\mathrm{a}>$ component is identified from I1- $\mathrm{N}_{\mathrm{b}}$ loop from Burgers circuit as compared with I1- $\mathrm{N}_{\mathrm{a}}$ type of dislocation loop. 

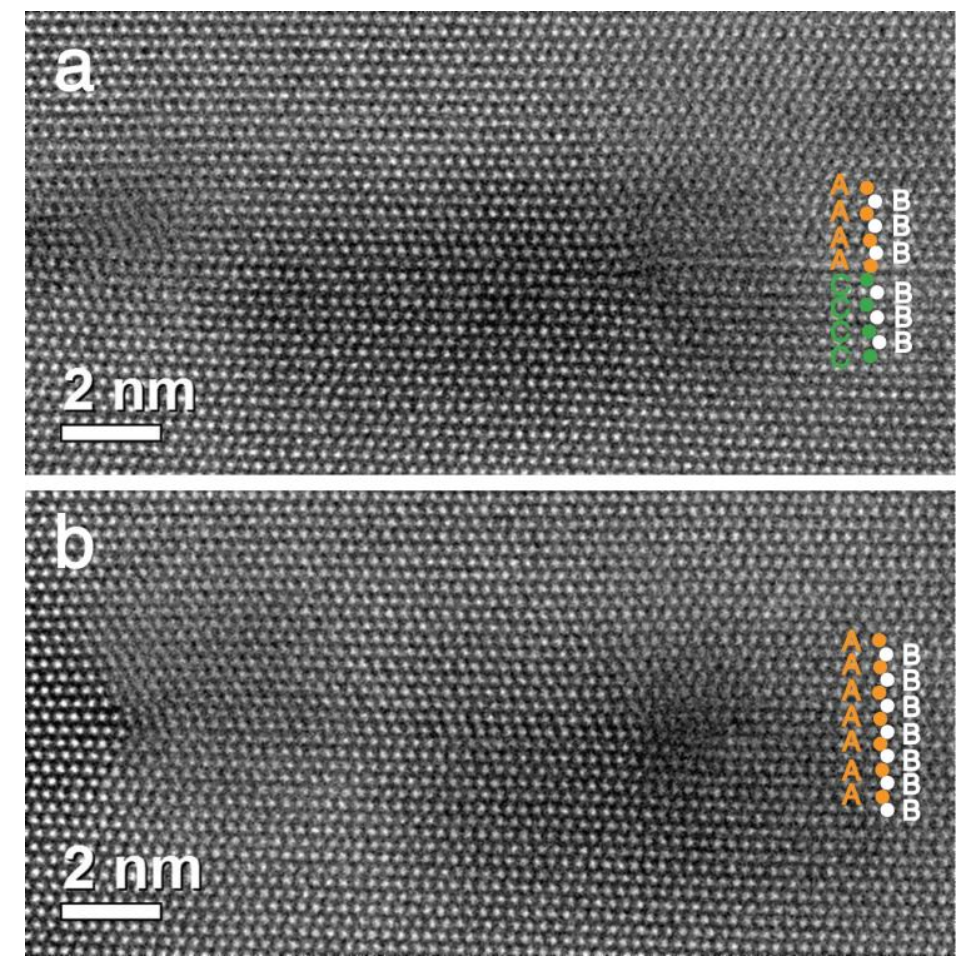

Figure 5. In situ observation of loop structure transformation from a), I1-I2 to b), I1-N type. The corresponding stacking sequences in the second layer loop region are marked in the figure.

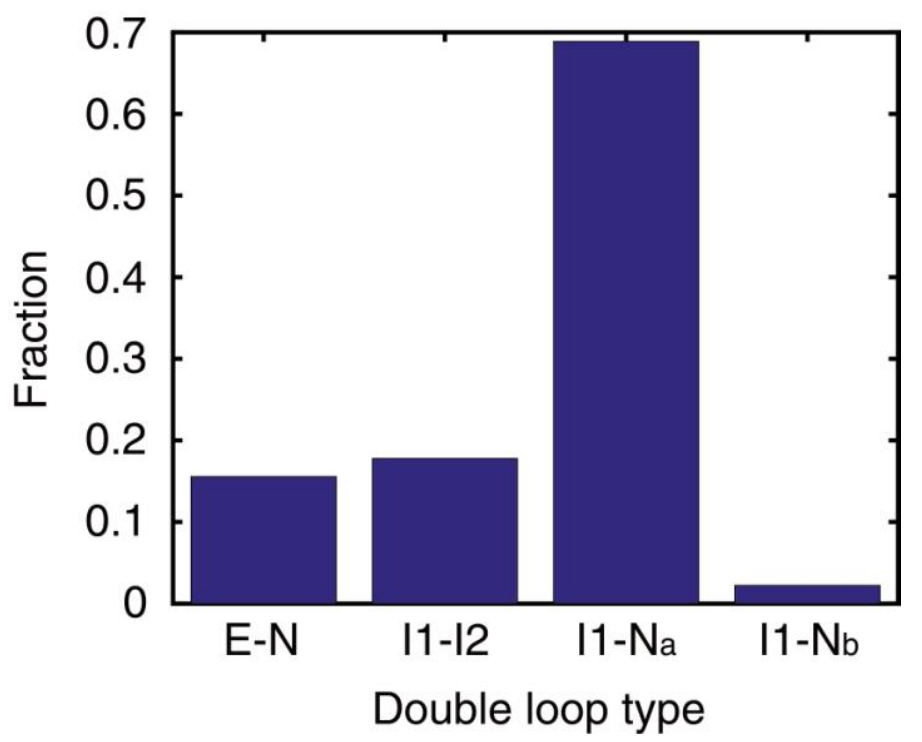

Figure 6. Relative abundance of the double-layer loop type from the STEM observation. 

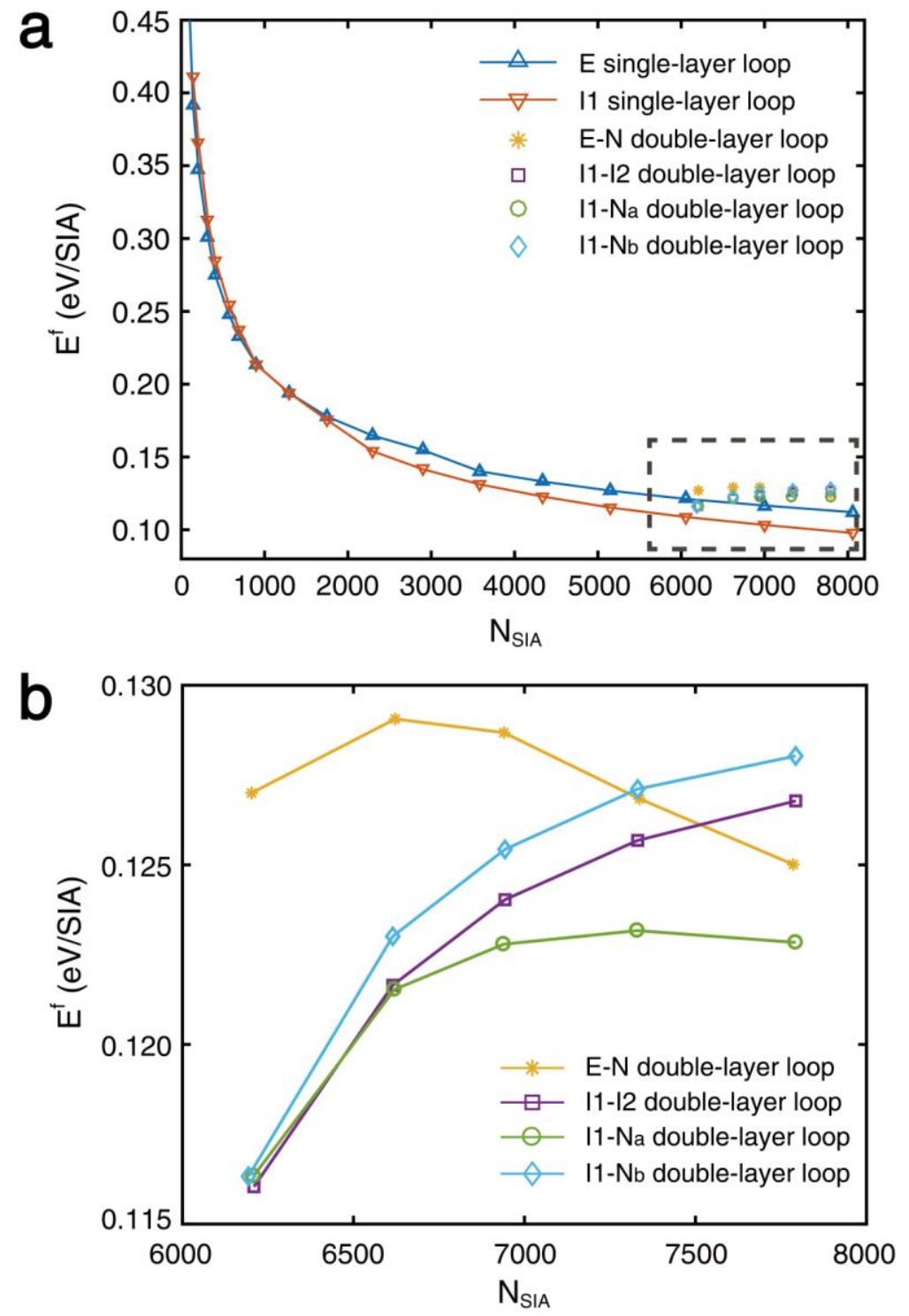

Figure 7. Specific formation energy $\left(E^{f}\right)$ of single- and double-layer loops calculated as a function of self-intestinal atom number in the loop. The enlarged area of Figure 7a marked by dash line is shown in Figure $7 b$. 


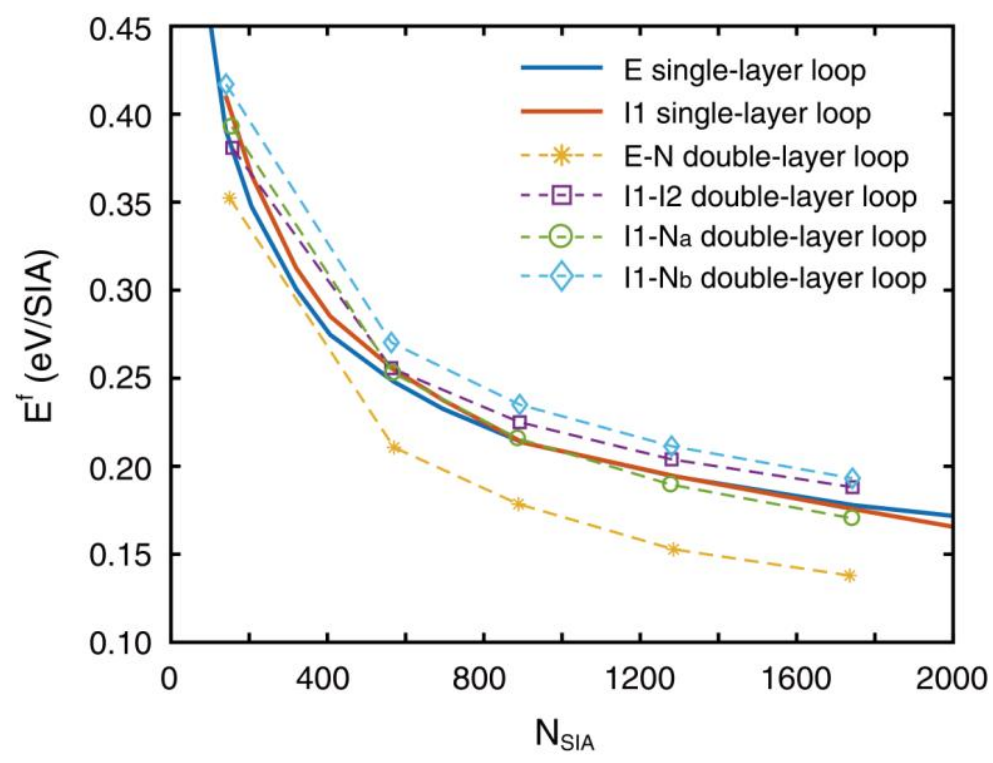

Figure 8. Specific formation energy of the second loop, $\mathrm{E}_{2}{ }^{\mathrm{f}}$, for different double-layer loop types, as compared with those of E and I1 single-layer loops.

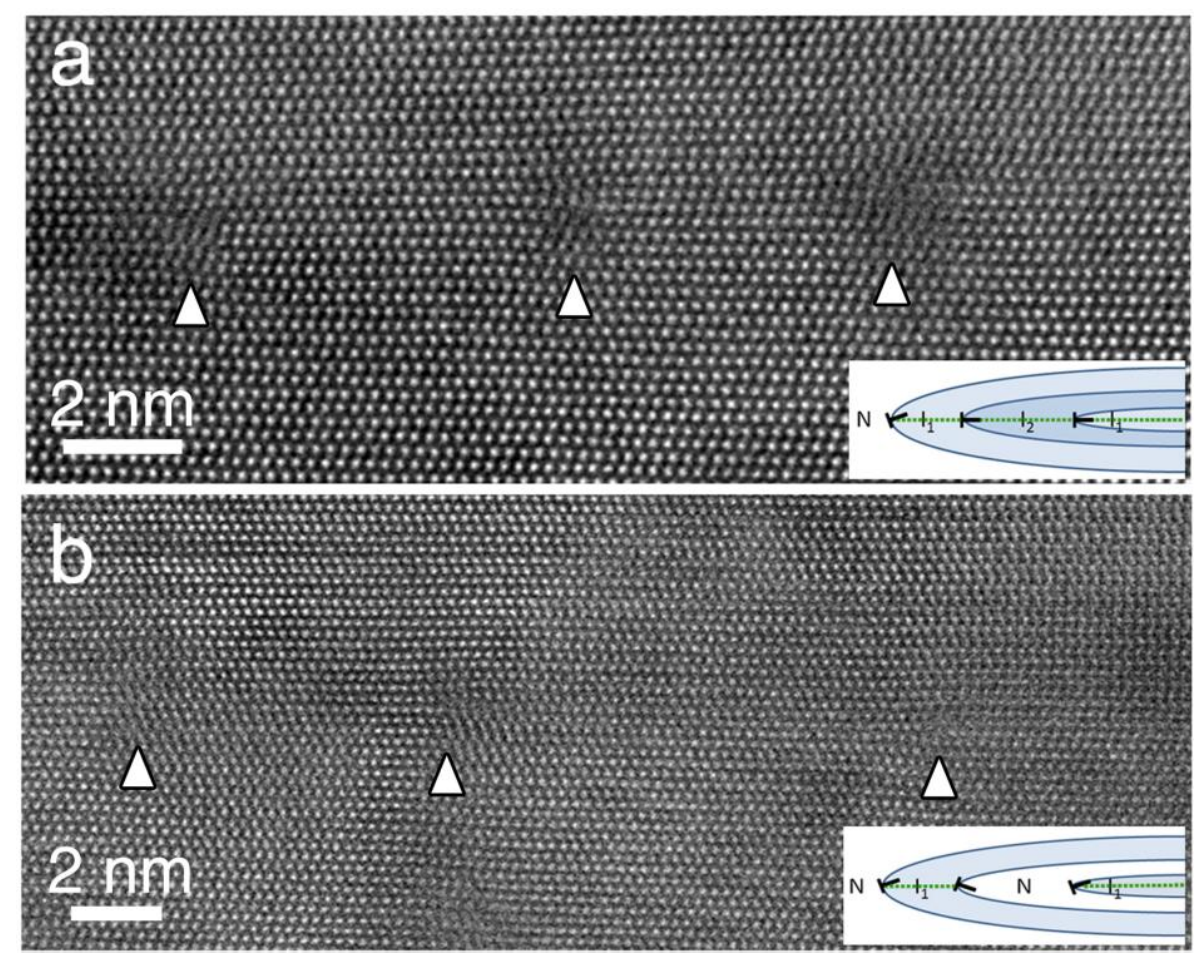

Figure 9. The observed triple loop structure by STEM, a, I1-I2-I1 type, b, I1-N-I1 type. The corresponding loop configuration is illustrated by the bottom-right corner insert. The loop edges were pointed out by arrows. 


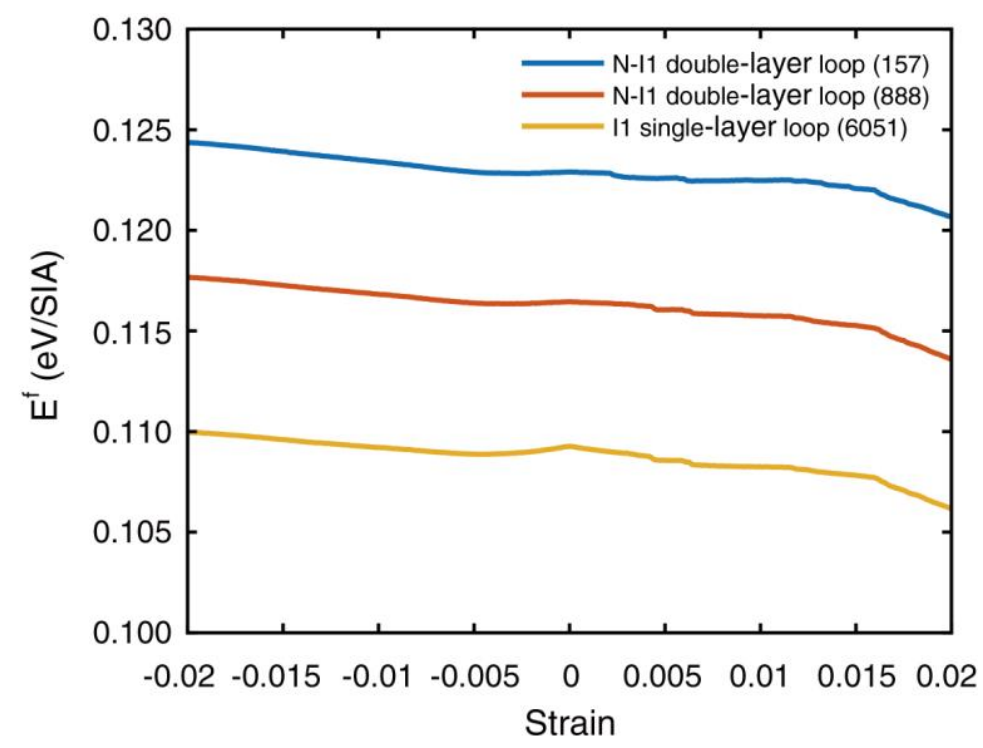

Figure 10. Specific formation energy of the N-I1 and single-layer I1 loops as a function of external strain along [0001] direction. Positive (negative) strain means tension (compression). 

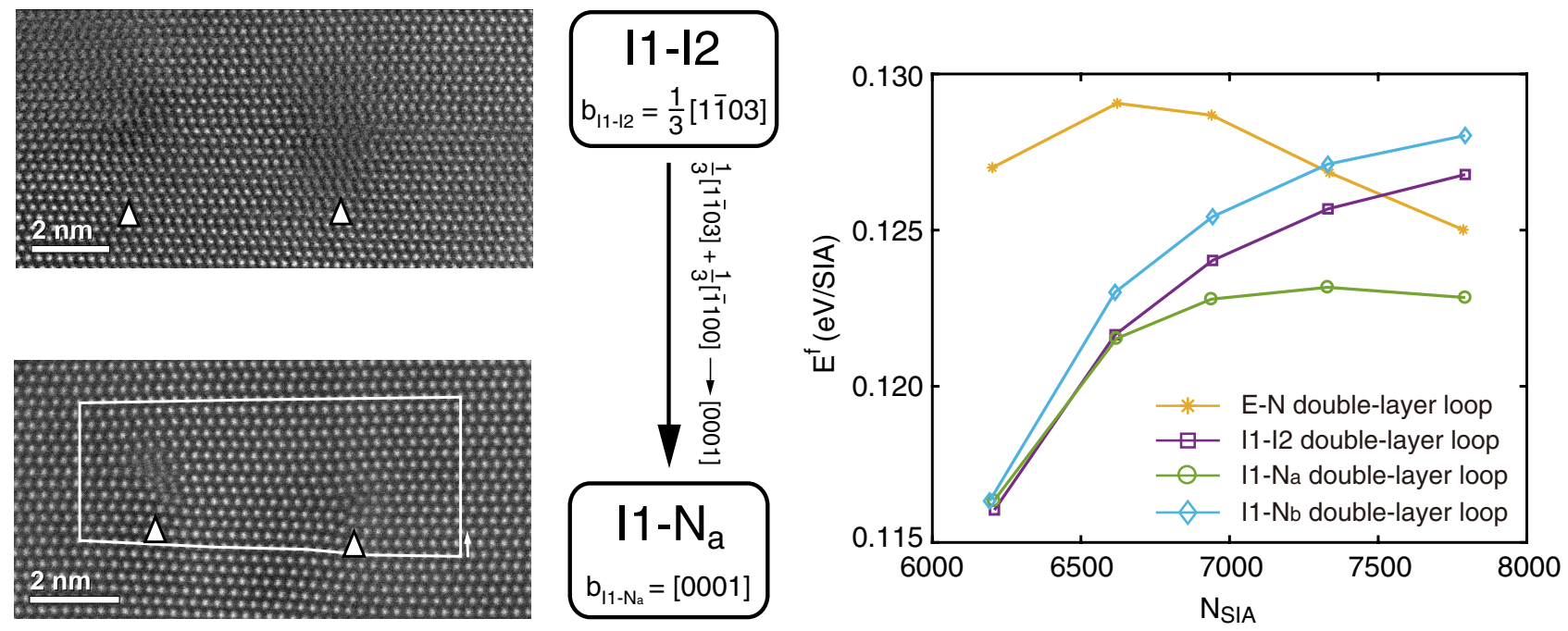